\section{Raucherentwöhnung in der primärärztlichen Praxis}

\author{
Einstellungen, Therapieerfahrungen und therapeutische Präferenzen \\ von Hausärzten
}

\author{
Stephan Mühlig' \\ Katja Hagenau ${ }^{1}$ \\ Eva Hoch ${ }^{2}$ \\ Holger Sonntag ${ }^{2}$ \\ Johannes Hacker ${ }^{1}$ \\ Michael Höfler ${ }^{2}$ \\ Hans-Ulrich Wittchen ${ }^{1,2}$
}

\author{
Smoking Cessation in Primary Care - Doctor's Attitude, Practical Knowledge and \\ Treatment Preferences in Germany
}

\section{Zusammenfassung}

Im Rahmen einer epidemiologischen Versorgungsstudie (SNICAS) wurden $n=872$ repräsentativ ausgewählte Hausärzte in Deutschland hinsichtlich ihrer Einstellungen, Therapieerfahrungen und therapeutischen Präferenzen gegenüber Raucherentwöhnungsangeboten in der Primärversorgung befragt. Trotz eines ausgeprägten Problembewusstseins werden Raucherentwöhnungstherapien von Hausärzten nur in Ausnahmefällen selbst durchgeführt. Während die meisten Ärzte ausreichende Kenntnisse und vereinzelte praktische Erfahrungen in der medikamentösen und nicht-medikamentösen Raucherentwöhnung besitzen, münden diese nur selten in Routineangebote für die Patienten. Die Gründe dafür aus Ärzteperspektive liegen vor allem in einer skeptischen Einschätzung der Patientenmotivation/-compliance und der Effektivität verfügbarer Therapieintervention sowie in versorgungspraktischen Hindernissen (Zeitaufwand, Abrechenbarkeit, unattraktive Vergütung). Darüber hinaus scheinen Selbstzweifel hinsichtlich der erforderlichen Kompetenzen auf Seiten der nicht-medikamentösen Interventionen (z. B. Psychoedukation, Beratung, Motivierung, Verhaltensinstruktionen) und strukturelle Versorgungsdefizite (fehlende Vernetzungsmöglichkeiten mit spezialisierten Einrichtungen und Therapeuten) eine Rolle zu spielen.

\section{Abstract}

In the context of an epidemiological final outcome study a representative sample of $n=872$ primary care doctors in Germany was investigated regarding their attitudes, prior experiences and personal preferences towards smoking cessation treatments in primary care. Despite doctors' high awareness of smoking as a core health care problem, they administer smoking cessation therapies very rarely. Most physicians show sufficient knowledge about smoking cessation and have sporadic prior practice in pharmacological or non-pharmacological first line treatments. However, they do not transfer their specific competencies into routine care. From the doctors' point of view the reasons for not providing such treatment are: patients' lack of motivation and compliance, doctors' scepticism with regard to the effectiveness of the available treatment options as well as practical problems (time exposure, payment). In addition, primary care doctors seem to be unconfident about their personal expertise in nonpharmacological interventions (i.e. patient-education, counseling, to motivate and instruct patients how to quit smoking). Furthermore structural deficits in German health care system play an important role.

Anmerkung

Die Durchführung der Studie Smoking and Nicotine Dependence Awareness and Screening (SNICAS) wurde durch einen unrestricted educational grant von GlaxoSmithKline (München) und GlaxoSmithKline-Consumer (Bühl) unterstützt. Die Studie ist Teil des Suchtforschungsverbundes ASAT (Allocating Substance Abuse Treatments to Patient Heterogeneity). (E-mail: asatkoordination@mpipsykl.mpg.de; www.asat-verbund.de) Der Forschungsverbund wird im Rahmen des Programms „Forschungsverbünde für Suchtforschung“ vom Bundesministerium für Bildung und Forschung finanziell gefördert (01 EB 0140-0142).

Institutsangaben

${ }^{1}$ Institut für Klinische Psychologie und Psychotherapie der Technischen Universität Dresden

${ }^{2}$ Max-Planck-Institut für Psychiatrie, Klinische Psychologie und Epidemiologie, München

Korrespondenzadresse

PD Dr. Stephan Mühlig · Technische Universität Dresden, Institut für Klinische Psychologie und Psychotherapie ·Chemnitzer Str. 46·01187 Dresden·E-mail: muehlig@psychologie.tu-dresden.de

Bibliografie

Suchttherapie 2003; Supplement 1, 4: S18-S25 @ Georg Thieme Verlag Stuttgart · New York · ISSN 1439-9903 
Schliisselwörter

Raucherentwöhnung · Primärversorgung · Epidemiologie · Versorgungsforschung
Key words

Smoking cessation $\cdot$ primary care $\cdot$ epidimiology $\cdot$ final outcome

\section{Einleitung}

Nach den Resultaten des BundesGesundheitssurveys rauchen trotz eines moderat rückläufigen Trends noch immer über 35\% der männlichen und knapp 27\% der weiblichen Bevölkerung in Deutschland regelmäßig (Zeitfenster: letzte 4 Wochen) Zigaretten bzw. andere Tabakprodukte [1-3]. Fast alle Raucher sind sich der rauchbedingten Gesundheitsrisiken bewusst, und ein Großteil unternimmt meist mehrfach den Versuch, damit aufzuhören [4]. Das BZgA [5] ermittelte, dass 35\% der rauchenden Männer und 33\% der rauchenden Frauen in den vorangegangenen 12 Monaten mindestens einen Aufhörversuch von mindestens einem Tag unternommen hatten. Allerdings enden eigenständige Aufhörversuche nur selten langfristig erfolgreich [6]. Die Abstinenzerwartung ohne Inanspruchnahme einer Therapie wurde in einer Metaanalyse bezogen auf die 12-Monats-Katamnese auf durchschnittlich $6,4 \%$ bestimmt [7]. Dennoch nehmen trotz der Verfügbarkeit effektiver Raucherentwöhnungstherapien [8-14] weniger als 5\% aller entwöhnungsbereiten Raucher professionelle Hilfsangebote in Anspruch [4].

Allerdings variieren auch die Erfolgsquoten von strukturierten Raucherentwöhnungsinterventionen sehr stark [15]. Der Einsatz von Selbsthilfemanualen erzielt mit 15\% Abstinenz im 12-Monats-Zeitraum nur unwesentlich bessere Ergebnisse als unsystematische Eigenversuche [16]. Nach einer Metaanalyse des US Department of Health and Human Services, die im Zusammenhang mit der Entwicklung der US Clinical Practice Guidelines durchgeführt wurde $[17,18]$, erreichen professionell geleitete bzw. medikamentös unterstützte Entwöhnungsmaßnahmen demgenüber weit höhere Erfolge in der 1-Jahres-Katamnese, z. B. verhaltenstherapeutische Gruppenprogramme (20-30\%), Nikotinersatzprodukte (21-27\%) und Bupropion $\left(Z^{\prime} \operatorname{ban}^{\circledR}, 23-38 \%\right)$. Diese Erfolgsquoten lassen sich durch Kombination von VT-Strategien und einer Nikotinsubstitution oder dem synchronen Einsatz zweier Substitutionsmethoden oder der Kombinationsbehandlung von Bupropion plus Nikotinersatz z. T. noch deutlich steigern (35-50\%) [19-31].

Trotz der herausragenden gesundheitspolitischen Bedeutung des Tabakrauchens [32-34] werden derartige professionelle Hilfsangebote zur Raucherentwöhnung in der Hausarztpraxis offenbar noch nicht hinreichend genutzt. Obwohl hierzu bislang aussagekräftige empirische Daten fehlen, wird aufgrund klinischer Erfahrungen vermutet, dass Raucherentwöhnungstherapien in der primärärztlichen Versorgungsrealität nur einen marginalen Stellenwert besitzen [35]. Dies ist insofern verwunderlich, als Hausärzte, praktische Ärzte und Internisten mit Primärarztfunktion potenziell eine zentrale Position für die Durchführung oder Vermittlung von Raucherentwöhnungsbehandlungen besitzen. Die 68000 Primärärzte spielen im deutschen Gesundheitssystem nicht nur eine Schlüsselrolle (,gate keeper“) bei der $\mathrm{Zu}$ gangssteuerung zu speziellen Versorgungsangeboten, sondern pflegen zudem meist einen langjährigen kontinuierlichen Kon- takt zu ihren Patienten, der ihnen eine Thematisierung der Problematik und das Anbieten von Raucherentwöhnungsmaßnahmen wesentlich erleichtern kann $[36,37]$. Wahrscheinlich wird dieses Einflusspotenzial im Rahmen der primärärztlichen Versorgung bislang eklatant unterschätzt [38]. Schon der ärztliche Rat, mit dem Rauchen aufzuhören, besitzt nach neueren Studienergebnissen eine wichtige Initialwirkung für die Rauchstoppmotivation, für Verhaltensänderungen (Entwöhnungsversuche) und die tatsächliche Rauchabstinenz ihrer Patienten [39]. Immerhin jedem zehnten Patienten gelingt der Zigarettenentzug allein aufgrund einer expliziten ärztlichen Empfehlung [40]. Auch Kurzberatungen durch den Hausarzt ( $<3$ Min.) können nach den Ergebnissen einer neueren Metaanalyse schon zu signifikanten Steigerungen der 12-Monats-Abstinenz auf 13,4\% (OR: 1.3; CI: 1.01-1.6) führen, die sich bei 3-10-minütiger Intervention auf $16,0 \%$ (OR: 1.6 ; CI: $1.2-2.0$ ) und bei über 10 Minuten Beratung sogar auf $22 \%$ (OR: 2.3; CI: 2.0-2.7) steigern lässt und damit fast den Wirkungsgrad verhaltenstherapeutischer Interventionen erreicht. Allerdings muss zu diesen Befunden einschränkend angemerkt werden, dass sie vorwiegend aus kontrollierten Wirksamkeitsstudien (efficacy) stammen und nicht unter realen Versorgungsbedingungen (effectiveness) gewonnen wurden.

Es stellt sich Frage, welche Gründe und Barrieren für die mangelnde Umsetzung der hausärztlichen Einflusspotenziale auf die Raucherentwöhnung von Patienten verantwortlich sind [41]. Da Indikations- und Interventionsentscheidungen des Arztes nicht nur von professionellem Handlungswissen, strukturellen Gegebenheiten und gesundheitspolitischen Rahmenbedingungen geprägt, sondern auch durch seine individuellen Einstellungsmuster und subjektiven Ansichten beeinflusst werden, wurden diese im Rahmen des SNICAS-Projekts explizit mit erhoben. In der Studie wurde im Einzelnen folgenden Fragestellungen nachgegangen:

- Welchen Stellenwert (subjektive Valenz) messen Hausärzte der Raucherentwöhnung a) generell in der allgemeinärztlichen Versorgung und b) für die eigene Praxis zu?

- Wie hoch ist der Informations- und Kenntnisstand der Hausärzte in Bezug auf die Problematik Tabakkonsum und -abhängigkeit, bestehende Hilfsangebote und die medikamentösen und nicht medikamentösen Interventionsmöglichkeiten?

- Mit welchen Therapie-/Interventionsverfahren bei der Tabakentwöhnung haben Hausärzte praktische Erfahrung? Welche werden von Ärzteseite als effektiv, welche als weniger nützlich beurteilt und welche in der praktischen Anwendung präferiert?

- Welche spezifischen Barrieren bestehen ggf. gegenüber dem Einsatz von Raucherentwöhnungstherapien in der eigenen Praxis?

- Hinsichtlich welcher Aspekte der Raucherentwöhnung besteht auf Ärzteseite besonderer Informations- und Unterstützungsbedarf? 
Die Studie Smoking and Nicotine Dependence Awareness and Screening (SNICAS) stellt eine zweistufig angelegte epidemiologische Versorgungsstudie zur Raucherentwöhnungstherapie in der hausärztlichen Praxis dar (vgl. Abb.1). Der erste Studienteil beinhaltete eine Punktprävalenzerhebung in Form einer repräsentativen Stichtagsbefragung, die im zweiten Abschnitt um eine randomisiert-kontrollierte Interventionsstudie im prospektiv-longitudinalen Design ergänzt wurde (zur detaillierten Beschreibung von Methodik und Design dieser weltweit größten Studie ihrer Art vgl. [42]). Zur Studiendurchführung wurden zunächst auf Grundlage einer bundesrepräsentativen Stichprobenziehung (1075 Hausarztpraxen) $n=889$ teilnahmebereite Hausärzte einer Vorbefragung mit einem ausführlichen Fragebogen („Praxiserhebungsbogen“) zu ihren Einstellungen, ihrer Behandlungspraxis und ihren Therapiepräferenzen in Bezug auf die Raucherentwöhnung unterzogen. Die Hausärzte wurden anschließend gebeten, alle an einem festgelegten Stichtag (7. Mai 2002) in der Praxis vorstelligen PatientInnen zu erfassen und nach ihrem Rauchstatus zu befragen ( $n=32$ 926; auswertbar: 28 707). Die identifizierten Raucher wurden dann mittels detaillierter Fragebogen sowie Arztratings genauer untersucht. Die hier präsentieren Ergebnisse basieren auf der Vorerhebung. Die zweite Phase (Interventionsstudie) wird an dieser Stelle nicht näher dargestellt.

Die Stichprobe setzt sich aus Ärzten der Fachrichtungen Allgemeinarzt (60,0\%), Internist $(24,6 \%)$ und praktischer Arzt $(15,6 \%)$ zusammen. Die einbezogenen Praxen verteilen sich geografisch relativ gleichmäßig auf Großstädte (37,7\%), Kleinstädte (30,3\%) und ländliche Gebiete (32,0\%), wobei die Regionen Dresden ( $n=97)$ und München $(n=80)$ mit zwei größeren separaten Substichproben vertreten waren, welche für die anschließende Interventionsstudie vorgesehen waren.

\begin{tabular}{|c|c|}
\hline \multicolumn{2}{|c|}{ Phase I (Epidemiologie) } \\
\hline \multicolumn{2}{|c|}{$\begin{array}{l}\text { Bundesweite repräsentative Arztauswahl } \\
\text { (1075 Ärzte) }\end{array}$} \\
\hline \multicolumn{2}{|c|}{$\downarrow$} \\
\hline \multicolumn{2}{|c|}{$\begin{array}{l}\text { Vorstudie (Praxisbogen; n= } \mathbf{8 7 2} \text { Praxen) } \\
\text { (Größe der Praxis, Qualifikation u. Einstellungen d. Arztes, } \\
\text { Patientenbeschreibung, Wissen, Prävalenzschätzungen) }\end{array}$} \\
\hline \multicolumn{2}{|c|}{$\downarrow$} \\
\hline \multicolumn{2}{|c|}{$\begin{array}{c}\text { Zieltag-Befragung Patienten ( } \mathbf{n = 3 2} \text { 926) bei } 889 \text { Ärzten } \\
\text { (Prävalenz des Rauchens nach Patient u. Arzt, Menge u. Häufigkeit, } \\
\text { Verhaltens- u. Risikoprofil, bisherige Behandlungen, Bereitschaft zun } \\
\text { Rauchstopp) }\end{array}$} \\
\hline \multicolumn{2}{|c|}{$\downarrow$} \\
\hline $\begin{array}{l}\text { Patienten-Fragebogen } \\
\text { (Soziodemographie, Rauchen, } \\
\text { Gesundheit, Nikotinabhängigkeit, } \\
\text { Motivation, frühere Rauchstopps) }\end{array}$ & $\begin{array}{c}\text { Arzt-Fragebogen } \\
\text { (Erkennung, klinische Risiken } \\
\text { und Schwere, Motivation u. } \\
\text { Behandlung) }\end{array}$ \\
\hline$\nabla$ & \\
\hline $\begin{array}{c}\text { Analyse der Ergebnisse } \\
\mathrm{n}=28707 \text { auswertbare } \\
\text { Datensätze von } 813 \text { hausärztlichen } \\
\text { Praxen }\end{array}$ & $\begin{array}{l}\text { Auswahl von Patienten für } \\
\text { Interventions-Phase II } \\
\text { Region Sachsen u. } \\
\text { Stadt/Land München }\end{array}$ \\
\hline
\end{tabular}

Abb. 1 Design und Methodik der SNICAS-Studie (erster Abschnitt).
Einstellungen und generelle Informiertheit der Studienärzte zur Raucherentwöhnung

\section{Relevanzbeurteilung der Problematik}

Raucherentwöhnung (RE) besitzt für die meisten Primärärzte offenbar eine große Bedeutung: Fast neun Zehntel $(89,5 \%)$ der Befragten halten diese für eher wichtig (59,9\%) oder sehr wichtig (29,6\%). Nahezu identisch wird die Bedeutung der RE in Bezug auf die Implementierung bzw. den Ausbau entsprechender Angebote für die eigene hausärztliche Praxis beurteilt (eher wichtig: 60,8\%; sehr wichtig: 23,1\%). In krassem Gegensatz zu diesem hohen Stellenwert, den die meisten der befragten Primärärzte dem Problem zuschreiben, schätzt eine knappe Zweidittelmehrheit $(62,9 \%)$ die konkreten Interventionsmöglichkeiten für die RE in der hausärztlichen Praxis schlecht ein (vgl. Abb. 2). Dabei werden mangelnde Motivation und Compliance der Patienten nahezu einhellig als wichtigste Hürde für den Erfolg von RE-Maßnahmen betrachtet: Zum einen wird als Hauptproblem bei der RE fast ausnahmslos (96,9\%) die Non-Compliance der Patienten genannt (darunter 55,8\% volle Zustimmung). Umgekehrt konstatieren fast alle Befragten (95,1\%), dass motivierten Patienten die RE auch ohne ärztliche Hilfe gelinge. Andererseits unterstützt aber nur jeder dritte Arzt (37,4\%) die Auffassung, dass Raucher

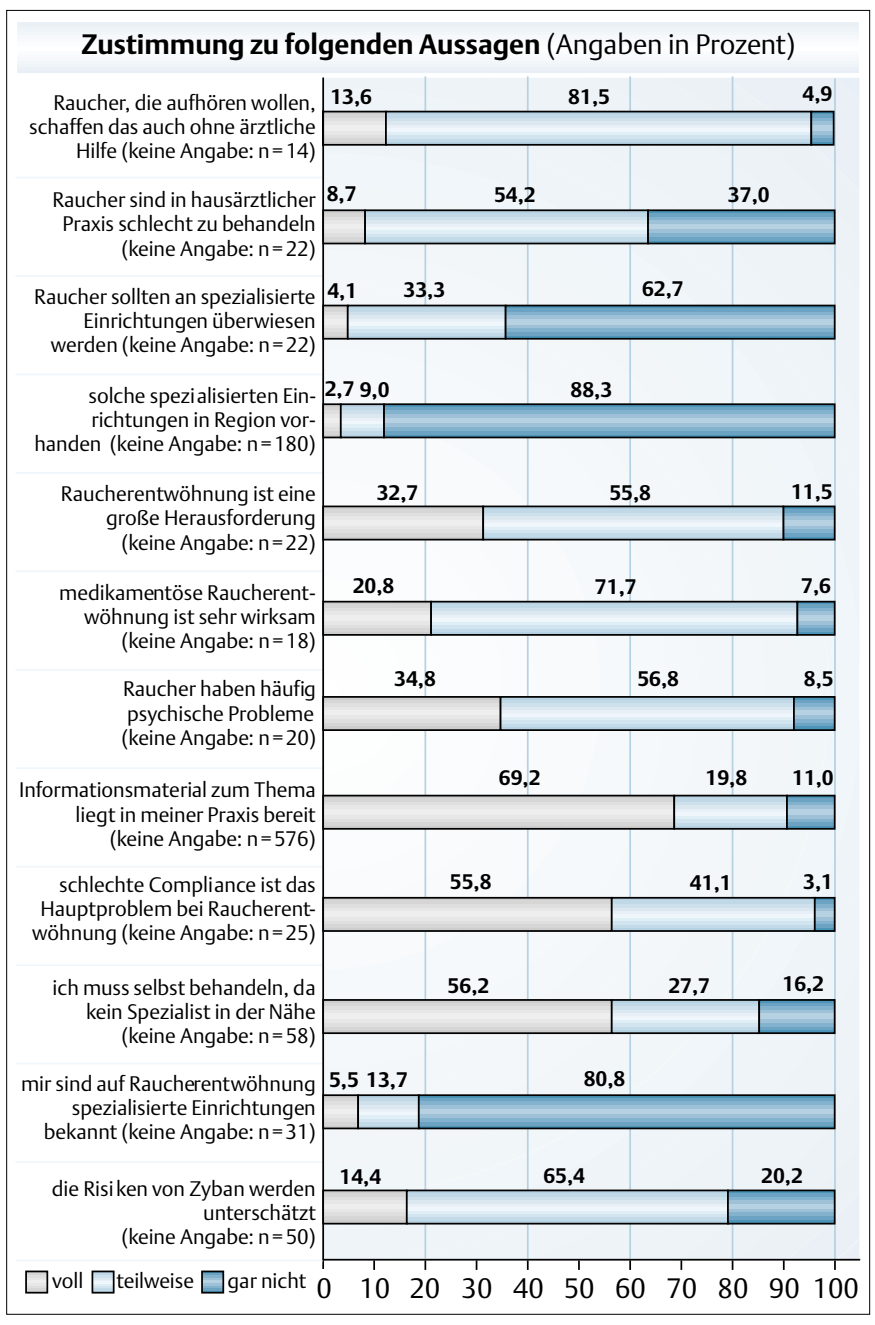

Abb. 2 Einstellungen der Hausärzte zu ausgewählten Aussagen zur Raucherentwöhnung. 
an spezialisierte Einrichtungen überwiesen werden sollten. Der überwältigenden Mehrheit der Befragten sind derartige Einrichtungen dabei entweder nicht bekannt $(80,8 \%)$ oder sie glauben, diese wären in der Region nicht vorhanden $(88,3 \%)$, so dass über die Hälfte (56,2\%) voll und weitere $27,7 \%$ teilweise überzeugt sind, in dieser Hinsicht selbst handeln zu müssen. Zusammenfassend deuten diese Ergebnisse auf eine überwiegend pessimistische Einschätzung der professionellen Therapieangebote zur RE hin, da zugleich die eigenen Therapiemöglichkeiten als schlecht und spezialisierte Angebote als unbekannt oder nicht verfügbar wahrgenommen werden.

\section{Beurteilung der Wichtigkeit spezifischer ärztlicher Fähigkeiten und Fertigkeiten für die Raucherentwöhnung}

Die teilnehmenden Ärzte wurden um eine Einschätzung der Wichtigkeit einer Reihe von spezifischen Fähigkeiten und Fertigkeiten für die RE in der hausärztlichen Praxis gebeten, deren Ergebnisse in Abb. 3 zusammengefasst sind.

Es zeigt sich, dass der Motivierung zum Rauchstopp der mit Abstand höchste Stellenwert beigemessen wird: 80,6\% der befragten Ärzte urteilen hier mit „sehr wichtig“ (weitere 18,3\% mit „eher wichtig“). Unter den nicht-medikamentösen Interventionen rangieren die Fähigkeiten für konkrete Verhaltensanweisungen (,sehr wichtig“: 60,1\%; „eher wichtig“: 34,6\%), den Einsatz verhaltenstherapeutischer Strategien („sehr wichtig“: 55,6\%;

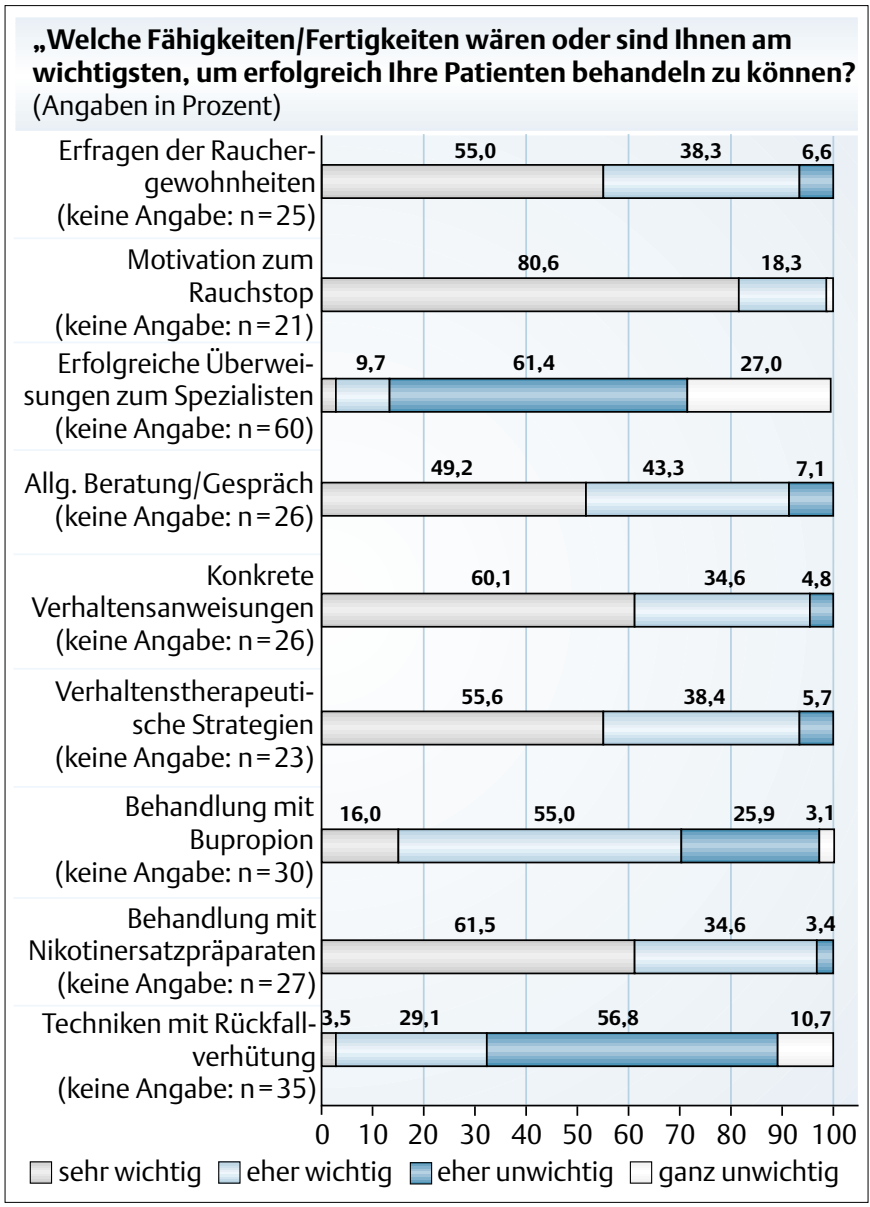

Abb. 3 Beurteilung der Wichtigkeit spezifischer Fähigkeiten und Fertigkeiten bei der Raucherentwöhnung. „eher wichtig“: $38,4 \%$ ) und das Erfragen der Rauchgewohnheiten („sehr wichtig“: 55,0\%; „eher wichtig“: $38,3 \%$ ) auf den mittleren Plätzen. Etwas geringer wird die Relevanz der kommunikativen Kompetenzen für allgemeine Beratung/ärztliche Gespräche („sehr wichtig“: 49,2\%; „eher wichtig“: 43,3\%) eingestuft. Demgegenüber werden ärztliche skills für den Einsatz von Techniken zur Rückfallprophylaxe nur von einer Minderheit als relevant angesehen („sehr wichtig“: 8,5\%; „,eher wichtig“: 29,1\%; „,eher unwichtig“: 56,8\%; „ganz unwichtig“: $10,7 \%$ ). Ähnlich verhält es sich mit dem Aspekt erfolgreiche Überweisung zum Spezialisten, der von über einem Viertel der Befragten als völlig und von weiteren zwei Dritteln als eher unwichtig beurteilt wird (,sehr wichtig“: 2,0\%; „eher wichtig“: 9,7\%; „eher unwichtig“: 61,4\%; „ganz unwichtig“: 27,0\%).

Bezogen auf die pharmakologischen Interventionsmöglichkeiten wird den ärztlichen Fertigkeiten für die Behandlung mit Nikotinersatzpräparaten („sehr wichtig“: 61,5\%; „eher wichtig“: 34,6\%) ein weitaus größerer Stellenwert eingeräumt als bei der Anwendung von Bupropion („sehr wichtig“: 16,9\%; „eher wichtig“: $55,0 \%$. Dies deutet darauf hin, dass beim Einsatz von Nikotinsubstitutiva weitaus größere Handhabungsprobleme und damit höhere Anforderungen an die ärztlichen Fähigkeiten wahrgenommen werden als bei der Anwendung des oralen Medikaments.

\section{Kenntnis und Einschätzungen der Wirksamkeit von pharmakologischen Raucherentwöhnungsinterventionen}

Insgesamt haben fast alle Ärzte (95,7\%) nach Selbstangaben bereits Erfahrungen mit Arzneimitteln zur Raucherentwöhnung sammeln können. Im Einzelnen wurde nach der Bekanntheit, bisherigen praktischen Erfahrungen sowie der eingeschätzten Wirksamkeit von drei Nikotinpflasterpräparaten, zwei Nikotinkaugummiprodukten, einem Nikotinnasalspray sowie dem Entzugsmedikament Bupropion $\left(\right.$ Zyban $^{\circledR}$ ) gefragt. Dabei besitzt Bupropion den höchsten Bekanntheits- und Erfahrungsgrad (90,9\%), gefolgt von den Kaugummi- $(24,4 \%-67,3 \%)$ und Pflasterprodukten (66,3\%-73,5\%). Am wenigsten Erfahrungen bestehen schließlich mit dem Nasenspray, das von weniger als einem Drittel (29,2\%) der Ärzte ausprobiert worden und immerhin einem weiteren Drittel überhaupt nicht bekannt ist (vgl. Abb. 4).

Wie in Abb. 2 ersichtlich, beurteilen fast alle befragten Ärzte die medikamentösen Möglichkeiten zur RE generell als „sehr effektiv" (volle Zustimmung: 20,8\%, teilweise: 71,7\%). Die Wirksamkeitsbeurteilung der einzelnen Präparate durch jeweils nur diejenigen Ärzte, die selbst praktische Erfahrung damit gesammelt hatten, fällt dabei aber sehr differenziert aus (vgl. Abb. 4): Die mit Abstand größte Effektivität wird der pharmakologischen Behandlung der Nikotinentzugssymptomatik mittels Bupropion zugeschrieben, das von der großen Mehrheit (85,1\%) der Ärzte als „eher gut geeignet“ eingestuft wurde. (Zugleich stimmen allerdings auch 93,8\% der Aussage zu, dass die Risiken von Bupropion unterschätzt würden.) Demgegenüber zeigt sich nur jeder dritte Hausarzt von der Eignung der Nikotinsubstitution mittels Pflastern (24,6\%-37,7\%) und und sogar nur ein Fünftel mittels Kaugummis (18,2\%-20,5\%) überzeugt. Das Nasalspray wird noch deutlich skeptischer beurteilt: Nur 16,4\% der Ärzte, die dieses Präparat bereits verordnet hatten, halten es für geeignet. 
Anzahl der Ärzte, die Erfahrungen mit Präparaten zur Raucherentwöhnung haben.

Unter diesen Ärzten Anzahl derjenigen, die das Präparat als „eher gut geeignet“ einschätzen (Angaben in absoluten Zahlen)

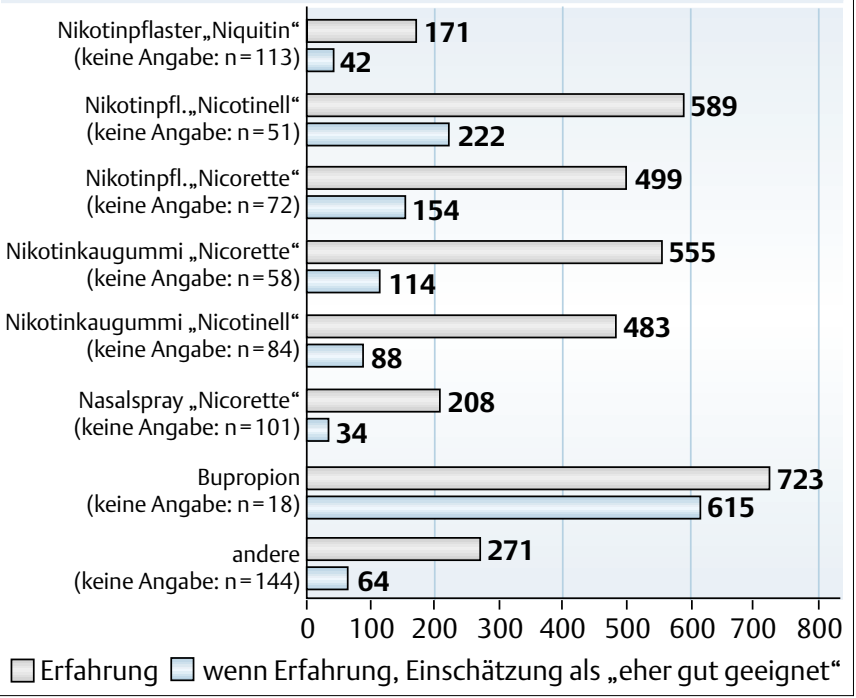

Abb. 4 Erfahrungen der Studienärzte mit unterschiedlichen Präparaten zur Raucherentwöhnung und Einschätzung ihrer therapeutischen Eignung (in absoluten Zahlen).

Zusätzlich wurden die ehemaligen bzw. aktiven Raucher unter den Ärzten danach gefragt, welche RE-Strategien sie persönlich bei ihrer eigenen Entwöhnung als hilfreich erlebt hatten. Unter den teilnehmenden Hausärzten war zum Zeitpunkt der Befragung mehr als die Hälfte (54,6\%) selbst Nichtraucher, fast ein Drittel (31,9\%) Exraucher und 11,9\% aktive Raucher. Unter den 346 Ärzten mit Rauchererfahrung (,Jemalsraucher“) hatten es zum Befragungszeitpunkt ca. drei Viertel geschafft, mit dem Rauchen aufzuhören. Knapp ein Drittel (30,1\%) aller Ärzte mit Rauchergeschichte gab an, „einfach aufgehört“ zu haben, während 63,9\% dafür hilfreiche Strategien oder Bedingungen benennen konnten: Unter diesen dominierte mit weitem Abstand der „starke Wille“ (42,5\%), während Rat und Unterstützung demgegenüber nur von $11,9 \%$ der nikotinerfahrenen Ärzte als nützlich beurteilt wurden. Medikamentöse RE-Interventionen verordneten sich die Ärzte selbst lediglich in Ausnahmefällen: Nur jeder 13. aufhörwillige Arzt (7,8\%) hat selbst Bupropion zum Nikotinentzug eingesetzt und sogar nur halb so viele (3,8\%) Nikotinsubstitution. Ebenfalls nur sehr wenige Ärzte (3,8\%) haben für die eigene Raucherentwöhnung selbst an einem Kursprogramm teilgenommen oder Selbsthilfebücher (4,3\%) benutzt.

\section{Barrieren und Unterstützugsbedarf gegenüber Raucherent- wöhnungsmaßnahmen Wahrgenommene Hinderungsgründe}

Aus gesundheitspolitischer Perspektive interessierten besonders die „Barrieren“, die Ärzte davon abhalten, RE-Therapien im Rahmen der Primärversorgung anzubieten. Die von den Befragten genannten Gründe sind in Abb. 5 zusammengestellt (Mehrfachnennungen möglich).

Konsistent zu den eingangs dargestellten subjektiven Ansichten der Ärzte wird die mangelnde Patientenmotivation als Hauptbarriere gegenüber RE-Maßnahmen genannt (55,4\%). An zweiter

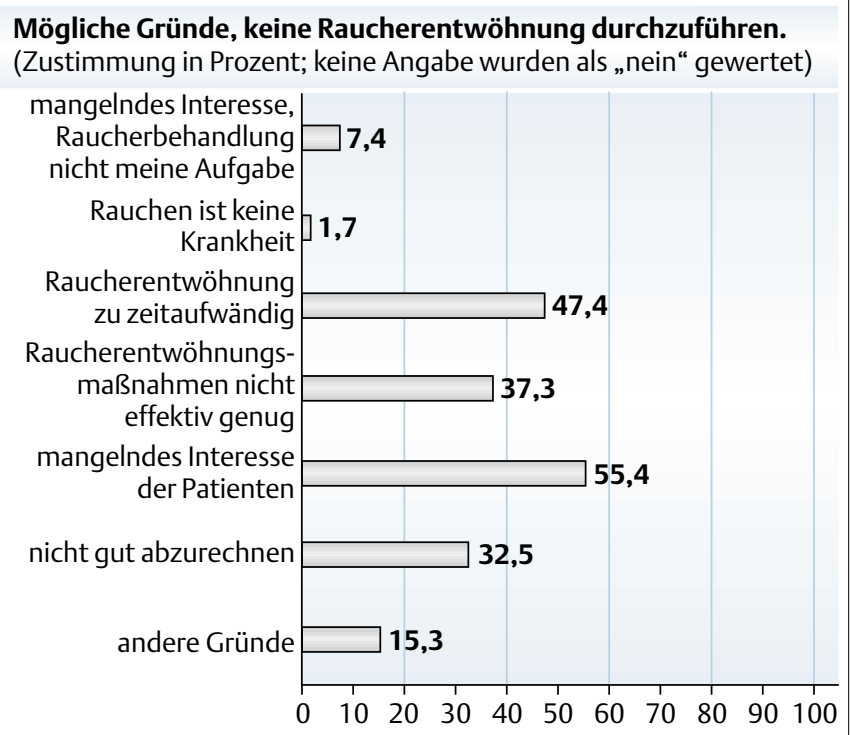

Abb. 5 Perzipierte Barrieren gegenüber Raucherentwöhnungsmaßnahmen in der allgemeinärztlichen Praxis.

Stelle stehen praktische Gründe: Fast jeder zweite Hausarzt (47,4\%) beurteilt die Raucherentwöhnungen als „zu zeitaufwändig“. Außerdem bildet für jeden dritten Hausarzt (32,5\%) die mangelnde Abrechenbarkeit einen gravierenden Grund, keine RE anzubieten. Explizite Ablehnung (,ist nicht meine Aufgabe“) findet sich nur in 7,4\% der Fälle. Überraschenderweise bewertet zudem über ein Drittel (37,3\%) der Ärzte RE-Maßnahmen als „nicht effektiv genug“, um sie in der eigenen Praxis einzusetzen. Dies steht offensichtlich in Widerspruch nicht nur zu der objektiven empirischen Befundlage, sondern auch zu der oben berichteten hohen generellen Effektivitätsbeurteilung durch die befragten Ärzte, insbesondere bzgl. der medikamentösen Verfahren.

\section{Wunsch nach Hilfe und Unterstützung bei der Raucherentwöhnung}

Als weitere mögliche Ursache für den unzureichenden Einsatz von RE-Maßnahmen kommen Unsicherheiten bzw. fehlende Kenntnisse in Bezug auf spezifische Interventionsstrategien infrage. Deshalb wurden die Studienärzte befragt, in welchen Bereichen sie sich besondere Hilfe und Unterstützung für ihre Praxis wünschen (vgl. Abb. 6).

Der größte Informations- bzw. Unterstützungsbedarf besteht interessanterweise bzgl. der Behandlung mit Nikotinersatzpräparaten. Nahezu jeder zweite Hausarzt (44,5\%) fühlt sich offenbar bei der Anwendung von Nikotinkaugummis, -pflastern oder -nasensprays unsicher oder überfordert. Jeder vierte Arzt (26,2\%) äußert demgegenüber den Wunsch nach Unterstützung hinsichtlich des Einsatzes von Bupropion. Obwohl mit weit größeren Nebenwirkungsrisiken behaftet, besteht offenbar weit weniger Informations- und Unterstützungsbedarf bei der Anwendung des oralen Medikaments als bei den technisch komplizierteren Applikationsformen der Nikotinersatzpräparate.

Standardisierte Kurzfragebogen und Screeninginstrumente zum Rauchverhalten sind vielen Ärzten nicht ausreichend bekannt oder verfügbar. Obwohl das Erfragen der Rauchgewohnheiten im 
Bereiche der Raucherentwöhnung, in denen weitergehende Hilfe erwünscht ist.

(Zustimmung in Prozent; keine Angabe wurden als „nein“ gewertet, wenn mindestens zu einem Item eine Antwort vorlag)

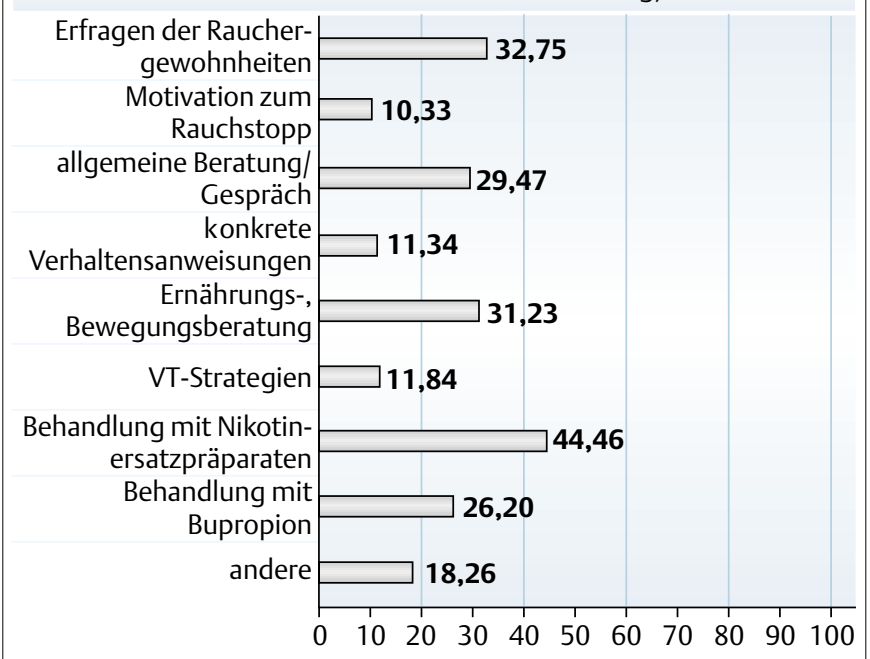

Abb. 6 Bereiche, für die spezielle Hilfe bei der Raucherentwöhnung artikuliert werden.

Vergleich zu den anderen Strategien relativ häufig durchgeführt wird (s. o.), wünscht sich jeder dritte Hausarzt (32,6\%) hier weitere unterstützende Maßnahmen. Ähnliches gilt für die Ernährungs- und Bewegungsberatung (31,2\%) und die allgemeine Beratung bzw. ärztliche Gesprächsführung (29,5\%). Diese Ergebnisse deuten darauf hin, dass besonders hinsichtlich der kommunikativen Kompetenzen auf Ärzteseite noch Fort- und Weiterbildungsbedarf besteht. Dies wird bestätigt durch die Quote von immerhin 67,1\% der Hausärzte, die gerne an einer Schulung zum „Motivational Interviewing“ teilnehmen würden. Überraschenderweise der geringste Unterstützungsbedarf wird in Bezug auf die psychologisch anspruchsvolleren Interventionen $V T$ Strategien (11,8\%), konkrete Verhalteninstruktionen (11,3\%) und Motivation zum Rauchstopp (10,3\%) artikuliert.

\section{Zusammenfassung und Diskussion}

Bilanzierend ist auf der Grundlage der Auswertungsergebnisse der Praxisvorerhebung der SNICAS-Studie festzustellen, dass die befragten Primärärzte die Relevanz von Raucherentwöhnungsbehandlungen hoch einschätzen, diese gleichzeitig aber relativ selten durchführen. Ein Grund für diese Diskrepanz zwischen ausgeprägtem Problembewusstsein einerseits und mangelnder Handlungsbereitschaft andererseits könnte in der überwiegend pessimistischen Einstellung der Ärzte gegenüber der Machbarkeit von RE-Therapien in der Praxisroutine bestehen, die von zwei Dritteln der Befragten geteilt wird. Offenbar wird das mittlerweile gut belegte Einflusspotenzial von Hausärzten auf die RE massiv unterschätzt. Den meisten Ärzten ist wahrscheinlich nicht bewusst, dass immerhin ein Teil ihrer rauchenden Patienten bereits durch Minimalinterventionen (ärztliche Empfehlung, 3-Minuten-Beratung) zum Rauchstopp bewegt werden könnte. In diesem Zusammenhang überraschen allerdings die Ergebnisse bezüglich der Überweisungpraxis an spezialisierte Kollegen oder Einrichtungen: Trotz der in der Wahrnehmung der Hausärzte be- grenzten Möglichkeiten zur RE in der eigenen Praxis ist nur gut ein Drittel (37\%) der Ansicht, dass entwöhnungsbereite Raucher an spezialisierte Kollegen oder Einrichtungen überwiesen werden sollten. Die Hauptursache dafür liegt wahrscheinlich in der geringen Versorgungsdichte bzw. fehlenden Informationen bezüglich spezialisierter Einrichtungen oder Therapeuten. Hier scheint ein eklatantes Versorgungs- und Informationsdefizit zu bestehen, da über $80 \%$ der Primärärzte keine spezialisierten REAnbieter in ihrer Region bekannt sind.

Das zentrale Problem für eine erfolgreiche RE liegt aus Ärzteperspektive in der mangelnden Patientenmotivation zum Rauchstopp. Fast ausnahmslos (97\%) wird hier die Non-Compliance der Patienten als Hauptproblem für eine erfolgreiche Entwöhnungsbehandlung benannt. Außerdem wird die unzureichende Motivation der Patienten als wichtigste Barriere für die Einführung von RE-Angeboten in der eigenen Praxis angesehen (55\%). Umgekehrt halten fast ebenso viele Ärzte eine RE auch ohne professionelle Hilfe für erfolgversprechend, sofern die Betroffenen nur ausreichend motiviert seien. Diese Einschätzungen widersprechen z. T. nicht nur der empirischen Befundlage, sondern offenbaren eine gravierende Unterschätzung des Abhängigkeitscharakters beim Tabakrauchen. Da jeder zweite regelmäßige Raucher die streng gefassten DSM-IV-Kriterien für Substanzabhängigkeit erfüllt (vgl. [43]), ist für viele Betroffene die Aufhörmotivation eine zwar notwendige, aber keineswegs hinreichende Bedingung für die langfristige Rauchabstinenz. Dies wird durch die klinische Evidenz für die drastische Überlegenheit professionell geleiteter RE-Maßnahmen bestätigt. In diesem Zusammenhang stimmt es nachdenklich, dass der überwiegenden Mehrheit der Hausärzte die internationalen Diagnosekriterien für eine Abhängigkeitsstörung nicht bekannt sind. Insbesondere hinsichtlich des Abhängigkeitsaspekts beim Tabakrauchen ist erheblicher Aufklärungsbedarf auf Ärzteseite zu konstatieren.

Die Motivierung zum Rauchstopp nimmt auch den höchsten Stellenwert bei der Beurteilung verschiedener Fähigkeiten und Fertigkeiten in der Raucherentwöhnung ein. Darüber hinaus wird vor allem den psychologisch-beraterischen Kompetenzen eine vergleichsweise hohe Bedeutung zugesprochen. Offenbar sind sich die meisten Ärzte bewusst, dass die RE in erster Linie durch professionelle Beratung und verhaltensnahe Interventionen erfolgen muss, die durch medikamentöse Maßnahmen wirksam unterstützt werden können. Inwieweit sich die Ärzte hinsichtlich der kommunikativen und psychologischen Anforderungen ausreichend kompetent fühlen, ist nicht eindeutig zu beurteilen. Diesbezüglichen Informations- und Unterstützungsbedarf äußert nur jeder dritte Befragte. Andererseits möchten aber über zwei Drittel an einer spezifischen Fortbildung zum „Motivational Interviewing“ teilnehmen.

Kenntnisse und partielle Erfahrungen in der RE-Therapie sind in der Ärzteschaft zwar weit verbreitet, münden aber nur selten in routinemäßige Versorgungsangebote im Rahmen der eigenen Praxis. Auf der einen Seite hat fast jeder Arzt (96\%) bereits Erfahrungen mit RE-Medikamenten gesammelt und beurteilt die medikamentösen Behandlungsmöglichkeiten generell als effektiv (93\%). Anderseits ist die Anzahl der im Einzelfall tatsächlich behandelten Patienten eher klein. Praktische Erfahrungen beim Einsatz von Nikotinersatzprodukten bestehen bei der Mehrheit 
der befragten Ärzte (je nach Präparat: bei 24-74\%). Bezogen auf das letzte Quartal wurden diese aber nur von einem Drittel (33\%) auch verordnet. Bupropion ist sogar über $90 \%$ der Befragten bekannt und wurde von fast 70\% im letzten Quartal eingesetzt (allerdings nur bei einer relativ kleinen Anzahl von Patienten). Die teilweise mäßige Effektivitätsbeurteilung der einzelnen RE-Methoden könnte einen weiteren Grund für deren mangelnde Verbreitung in der Versorgungspraxis darstellen. Als sehr wirksam (85\%) wird allein die Bupropion-Behandlung beurteilt, die allerdings aus unterschiedlichen Gründen (Kontraindikationen, unerwünschte Arzneimittelwirkungen und -risiken, Preis) für viele Patienten nicht infrage kommt. Weitaus skeptischer fallen die Beurteilungen gegenüber den Nikotinsubstitutionspräparaten aus. Nur jeder dritte bis fünfte Arzt, der bereits Erfahrungen mit Nikotinpflastern oder-kaugummis gesammelt hat, ist von deren Nützlichkeit überzeugt (beim Nasalspray sogar nur 16\%). Möglicherweise ist diese Haltung in erster Linie auf Anwendungsprobleme zurückzuführen. Den Kompetenzen beim Einsatz von $\mathrm{Ni}$ kotinersatzpräparaten wird vermutlich deshalb ein vergleichsweise hoher Stellenwert zugesprochen, weil sie technisch nicht einfach zu handhaben sind und ihre Anwendung den Patienten relativ schwer zu erklären ist. Da hier auch der größte Informations- und Unterstützungsbedarf artikuliert wird, ist davon auszugehen, dass auch auf Ärzteseite einige Unsicherheit bei ihrer Anwendung besteht. Dies wird gestützt durch die relativ skeptische Beurteilung der Eignung von Substitutionspräparaten für die RE. Während vier von fünf Studienärzten, die bereits Erfahrungen mit Bupropion gemacht haben, sie als geeignet beurteilen, werden spezielle Kenntnisse oder Fertigkeiten bei dieser Form der medikamentösen Behandlung nur von 16\% der Befragten als relevant eingestuft. Dies ist vermutlich darauf zurückzuführen, dass die ärztlichen Anforderungen beim Einsatz eines oralen Medikaments als wesentlich geringer eingeschätzt werden als die für die Kaugummi- und Pflasterapplikationen erforderlichen Instruktionsfähigkeiten. Damit werden die von den Ärzten wahrgenommenen Kompetenzerfordernisse eher auf technische Handhabbarkeitsaspekte als auf die Wirkweise der Substanzen fokussiert, obwohl andererseits von über $90 \%$ der Befragten das Nebenwirkungsrisiko von Bupropion betont wird.

Neben der skeptischen Einschätzung der Praktikabilität von RETherapien in der eigenen Praxis (unzureichende Therapiemotivation der Patienten, geringe Erfolgserwartungen des Arztes an die verfügbaren Therapieoptionen) sind es aber auch strukturelle und logistische Rahmenbedingungen, die eine Ausweitung des Therapieangebots im primärärztlichen Sektor behindern. Für fast jeden zweiten Arzt (47\%) ist die RE in der Praxis zu zeitaufwändig, für jeden dritten (33\%) zu schlecht abzurechnen und für über $37 \%$ in der Eigenanwendung nicht effektiv genug. Angesichts der Tatsache, dass deutsche Hausärzte mit durchschnittlich 70 persönlichen Patientenkontakten pro Tag im europäischen Vergleich eine fast doppelt so hohe Patientenzahl versorgen wie ihre Kollegen in den Nachbarländern, verwundern die praktischen Probleme bei der Durchführung zeitaufwändiger patientennaher Interventionen wie der RE kaum. Wahrscheinlich lassen sich effektive RE-Angebote in der ambulanten Versorgung deshalb nur auf der Basis angemessener Fallpauschalen realisieren. Der Durchführung von RE-Therapien in der Hausarztpraxis stehen aber nicht nur diverse Einstellungsbarrieren und praktische Hindernisse entgegen, sondern auch Defizite bez. der erforderlichen Kompetenzen, vor allem auf Seiten der nicht medikamentösen Interventionen (z. B. Psychoedukation, Beratung, Motivierung, Verhaltensinstruktionen). Insbesondere die Vernetzung mit spezialisierten Einrichtungen und Therapeuten sowie die Verwendung internationaler Assessments zur Anhängigkeitsproblematik und die Orientierung an Therapieleitlinien zur RE scheinen dringend verbesserungsbedürftig.

\section{Literatur}

${ }^{1}$ Jacobi F, Wittchen HU, Hölting C et al. Estimating the prevalence of mental and somatic disorders in the community: aims and methods of the German National Health Interview and Examination Survey. Int J Methods Psychiatr Res 1998; 11: 1- 18

${ }^{2}$ Perkonigg A, Lieb R, Wittchen HU. Substance use, abuse and dependence in Germany: A review of selected epidemiological data. Eur Addiction Res 1998; 4 (1-2): 8-17

${ }^{3}$ Sonntag H, Pfister H, Wittchen HU. Rauchen und Nikotinabhängigkeit in Deutschland. Ergebnisse des bundesweiten Zusatz-Surveys „Psychische Störungen“ im Rahmen des Bundes-Gesundheitssurveys. 1998

${ }^{4}$ Nelson CB, Wittchen HU. Smoking and nicotine dependance: Results from a sample of 14-24-years olds in Germany. Eur Addiction Res 1998; $4: 42-49$

${ }^{5}$ Bundeszentrale für gesundheitliche Aufklärung (BzgA). Die Drogenaffinität Jugendlicher in der BRD - Wiederholungsbefragung 2001. Köln, 2001

${ }^{6}$ Jarvis MJ. Patterns and predictors of smoking cessation in the general population. In: Bolliger CT, Fagerström KO (Hrsg). The tobacco epidemic. Prog Resp Res 1997; 115-164

${ }^{7}$ Baillia AJ, Mattick RP, Hall W. Quitting smoking: estimation by metaanalysis of the rate of unaided smoking cessation. Aust J Public Health 1995; 19: 129-131

${ }^{8}$ Arzneimittelkommission der deutschen Ärzteschaft. Empfehlungen zur Therapie der Tabakabhängigkeit. Suchtmed 2001; 3: 156- 175

${ }^{9}$ Bühringer G, Körger C, Küfner H et al. Suchtforschungsverbund ASAT: Entwicklung von Zuordnungsmodellen für Interventionen bei Substanzstörungen. Sucht 2002; 48 (3): 200-208

${ }^{10}$ Arbeitskreis Rauchen und Gesundheit. Consensus Statement, 1998

${ }^{11}$ Bundesärztekammer/Kassenärztliche Bundesvereinigung. Frei von Tabak. Stufenprogramm zur Raucherberatung und Rauchertherapie in der Arztpraxis. Köln, 1997

${ }^{12}$ Deutsches Krebsforschungszentrum. Die Rauchersprechstunde - Beratungskonzept für Gesundheitsberufe. Heidelberg, 2000

${ }^{13}$ Kunze U, Schmeiser-Rieder A, Schoberberger R et al. European Medical Association Smoking for Health (EMASH) - Konsensuspapier Rauchertherapie: Richtlinien für Ärzte. Soz Präventivmed 1998; 43: 167-172

14 WHO. Evidence based Recommendations on the treatment of tobacco dependance. 2001 WHO-recommAug2001.pdf; www.who-nichtrauchertag.de

${ }^{15}$ Silagy C, Mant D, Fowler G et al. Meta-analysis on efficacy of nicotine replacement therapies in smoking cessation. Lancet 1994; 343: $139-142$

${ }^{16}$ Brown SL, Owen N. Self-help smoking cessation materials. Aust J Public Health 1992; 16: 188-191

17 US Department of Health and Human Services. People Health. National Health Promotion and Disease Prevention Objectives. Full Report. Washington 1990

${ }^{18}$ Fiore MC. US Public Health Practice Guideline: Treating tobacco use and dependence. Resp Care 2000; 45: 1200-1262

${ }^{19}$ American Psychiatric Association. Practice guideline for the treatment of patients with nicotine dependence. Am J Psychiatry 1996; 153 (suppl. 10): S1 -S31

20 Batra A, Buchkremer G. Raucherentwöhnung mit dem Nikotinpflaster. Z Ärztl Fortb 1995; 89: 505-509

${ }^{21}$ Buchkremer G, Bents H, Horstmann M et al. Combination of behavioral smoking cessation with transdermal nicotine substitutution. Addict Beh 1989; 14: 229-238

${ }^{22}$ Buchkremer G, Minneker E, Block M. Smoking-cessation treatment combining transdermal nicotine substitution with behavioral therapy. Pharmacopsychiatry 1991; 24: $96-102$ 
${ }^{23}$ Fagerström KO, Schneider NG, Lunell E. Effectiveness of nicotine patch and nicotine gum as individual versus combined treatment for tobacco withdrawal symptoms. Psychopharmacol 1993; 111: 271 - 277

24 Jorenby DE, Leischow JJ, Nidel MA et al. A controlled trial of substained-release Bupropion, a nicotine patch, or both for smoking cessation. N Engl J Med 1999; 340: 685-691

${ }^{25}$ Haustein KO. Pharmacotherapy of nicotine dependence. Int J Clin Pharmacol Ther 2000; 38: $273-290$

${ }^{26}$ Hurt RD, Sachs DP, Glover ED et al. A comparison of substained-release bupropion and placebo for smoking cessation. N Engl J Med 1997; 337: $1195-1202$

27 Prochaska JO, DiClemente CC, Velicer WF et al. Standardized, individualized, interactive, and personalized self-help programs for smoking cessation. Health Psychol 1993; 12: 399-405

${ }^{28}$ Schön S, Nowak D. Medikamentöse Verfahren zur Raucherentwöhnung. Suchtmed Forsch Prax 2002; 3 (Sonderdruck)

${ }^{29}$ Sonntag H, Kröger C, Shaw R. Setting up an information system on smoking cessation programmes. IFT Berichte, Bd. 96. München: IFT Institut für Therapieforschung 1998

${ }^{30}$ Wittchen HU, Sonntag H, Schmidt K. Rauchfrei in der 3-Punkt-Therapie. Selbsthilfemanual und Tagebuch zur Unterstützung der Tabakentwöhnung mit Zyban. Hamburg: Glaxo 2000

${ }^{31}$ Zimmer D, Lindinger P, Mitschele U. Neue Wege in der verhaltenstherapeutischen Behandlung des Rauchens. Verhaltensther 1993; 3: $304-311$

32 Sachverständigenrat für die konzertierte Aktion im Gesundheitswesen (SVR). Bedarfsgerechtigkeit und Wirtschaftlichkeit, Bd. III: Über-, Unter- und Fehlversorgung. 2000/2001; 139-149
${ }^{33}$ WHO. Tobacco or health: a global status report. Geneva: World Health Organization 1997

${ }^{34}$ WHO: Gemeinsam handeln - Tabakkonsum reduzieren: Dokumentation der Abschlusskonferenz des WHO-Partnerschaftsprojektes Tabakabhängigkeit (1999-2002). WHO-Konferenzband.pdf: www. who-nichtrauchertag.de. 2002

${ }^{35}$ Haustein K. Raucherentwöhnung: Primäre ärztliche Aufgabe. Deutsch Ärztebl 2002; 99: A-3162 - 3163

${ }^{36}$ Bellach BM, Knopf H, Thefeld W. Der Bundesgesundheitssurvey 1997/98. Gesundheitswesen 1998; 60 (Sonderheft 2): 59-68

37 Wittchen HU, Sonntag H. Raucherentwöhnung beim Hausarzt - Illusion oder Perspektive? Hausarzt 2002; 6: 49-53

${ }^{38}$ Fowler G. Smoking cessation: the role of general practitioners, nurses and pharmacists. In: Bolliger CT, Fagerström KO (Hrsg). The tobacco epidemic. Progress in respiratory research. Basel: Karger 1997; $165-177$

39 Junge B, Thamm M. Jahrbuch Sucht 2003, Tabak - Zahlen und Fakten zum Konsum (34-61). Geesthacht: Neuland-Verlag 2001

${ }^{40}$ Fiore MC, Bailey WC, Cohen SJ et al. Treating tobacco use and dependence. Clinical practice guideline. Rockville, MD: US Department of Health and Human Services. Public Health Services, 2000

${ }^{41}$ Ferry LH. Overcoming barriers to nicotine dependance treatment. Prim Care 1999; 26: 707

42 Muehlig S, Hoch E, Hoefler M et al. Design and Methods of SNICAS study. Int J Methods Psychiatr Res 2003 [accepted]

${ }^{43}$ Hoch E, Muehlig S, Hoefler M et al. Smoking and nicotine dependence among primary care attendees in Germany. Addiction [submitted]

\section{Suchttherapietage in Hamburg}

\section{Schwerpunktthema: \\ „Ansätze der Frühintervention zwischen Selbstmanagement und motivierender Gesprächsführung“}

1.-4. Juni 2004

Es geht bei jeder Störung darum, möglichst früh zu intervenieren und riskanten Entwicklungen, Chronifizierungen und Komplikationen vorzubeugen. Frühinterventionen können nur selektiv und auf kleine Gruppen beschränkt sein und müssen zwischen Prävention und klassischem Therapiesystem besondere Strategien entwickeln. Sie stellen ein notwendiges Bindeglied zwischen beiden Interventionsbereichen dar. Methoden der Selbstkontrolle und des Selbstmanagements sind ein gutes Beispiel, ergänzt durch eine konstruktive Nutzung des Internets und Ansätze motivierender Gesprächsführung. Wir wollen zur Verbreitung dieser positiven Erfahrungen beitragen.

\section{Veranstalter}

Zentrum für Interdisziplinäre Suchtforschung der Universität Hamburg (ZIS), Institut für interdisziplinäre Sucht- und Drogenforschung (ISD, Hamburg), Klinik und Poliklinik für Psychiatrie und Psychotherapie, Universitätsklinikum Hamburg-Eppendorf u.a.

\section{Weitere Informationen und das Programmheft erhalten Sie über das}

Kongressbüro „Suchttherapietage“, Zentrum für Interdisziplinäre Suchtforschung der Universität Hamburg, Klinik für Psychiatrie, UKE, Martinistr. 52, D-20246 Hamburg. Fax: 0 40/4 28 03-51 21

E-mail: kontakt@suchttherapietage.de 